\title{
Proficiency of Laryngeal Mask Airway Insertion Skill in NRP Certified Providers
}

\author{
Srinivasan Mani, MD ${ }^{1}$ Munmun Rawat, MD ${ }^{1}$ \\ ${ }^{1}$ Department of Pediatrics, University at Buffalo, Buffalo, New York \\ Am J Perinatol 2022;39:1008-1014.
}

Address for correspondence Srinivasan Mani, MD, Neonatal-Perinatal Medicine, Fellow Physician PGY-6, 1001 Main Street, 5th Floor, Buffalo, NY 14203 (e-mail: drvazan@gmail.com).

Abstract


- intratracheal
- resuscitation
- laryngeal mask airway
- infant, newborn
-

Objective In 2015, Neonatal Resuscitation Program (NRP) recommended laryngeal mask airway (LMA) as an alternative to the endotracheal tube (ETT) in situations where the provider is "unable to intubate and unable to ventilate." LMA insertion is being taught in the NRP routinely. However, endotracheal intubation is the primary method considered as the standard of care in neonatal resuscitation. LMA insertion is a relatively simple procedure with an average insertion time of $<10$ seconds. Newer generation LMA can have the added advantage of reducing the risk of aerosol generation and improving the safety of the providers. Only a few recent studies have evaluated the LMA insertion skills of neonatal resuscitation providers. We wanted to study the proficiency of NRP providers in the technique of LMA insertion. We hypothesized that NRP providers would have LMA insertion skills equivalent to the standard of care (ETT insertion).

Study Design A manikin-based study was done from July 2019 to December 2019. We enrolled 31 NRP providers with 1 or more years since the first certification and current valid NRP provider/instructor status. The participants were instructed to insert an ETT and LMA in the manikin. The procedures were video recorded. The time taken to insert and start ventilation with each device, including the number of attempts for successful insertion, was noted. A Likert scale questionnaire was filled by each participant indicating the level of confidence, perception of ease, and the ability to provide effective positive pressure ventilation (PPV) with each of the procedures. The paired $t$-test, chi-square test, and Kruskal-Wallis' test were used for the statistical analysis.

Results Eight (25.8\%) out of the 31 participants failed to insert any one of the devices. So, 23 providers were analyzed for the outcomes. We found that the mean duration taken to insert the ETT and LMA was not statistically different ( 32 vs. 36 seconds). LMA insertion had a higher failure rate compared with ETT. Providers did not perceive confidence to insert LMA when compared with ETT. They did not recognize LMA insertion as a more effortless procedure relative to endotracheal intubation. The providers felt that their ability to provide effective PPV using LMA was inferior to ETT. Conclusion The NRP certified providers in this study did not demonstrate proficiency in the insertion of LMA equivalent to the endotracheal intubation. received

September 15, 2020 accepted after revision October 16, 2020

published online

November 29, 2020
DOI https://doi.org/ 10.1055/s-0040-1721379. ISSN 0735-1631.

\footnotetext{
(C) 2020. The Author(s).

This is an open access article published by Thieme under the terms of the Creative Commons Attribution-NonDerivative-NonCommercial-License, permitting copying and reproduction so long as the original work is given appropriate credit. Contents may not be used for commercial purposes, or adapted, remixed, transformed or built upon. (https://creativecommons.org/ licenses/by-nc-nd/4.0/) Thieme Medical Publishers, Inc., 333 Seventh Avenue, 18th Floor, New York, NY 10001, USA
} 


\section{Key Points}

- LMA insertion skill was studied in NRP providers using a manikin.

- Providers had a higher frequency of failure in inserting LMA compared to ETT.

- Providers' perceived confidence and effectiveness of the LMA procedure were inferior to ETT.

Provider preparedness in psychomotor skills such as securing a patent airway plays a critical role in the delivery of neonatal resuscitative efforts. Skill readiness in providers on low frequency, high-impact emergency procedures is dependent on structured training such as newborn resuscitation programs ${ }^{1}$ and opportunities to practice the learned skill regularly within the scope of clinical practice. Studies have shown that skill decay would happen in providers within 6 months after initial training without regular training refreshments. ${ }^{2}$

Endotracheal tube (ETT) placement is an intricate emergency procedure in critical care medicine, including neonatal intensive care. Studies have reported first attempt success rates $<50 \%$ for tracheal intubation (TI) in neonates. ${ }^{3,4}$ Successful attempts frequently take $>30$ seconds. In one earlier study, success rates and mean (standard deviation [SD]) time to intubate successfully varied based on the provider's experience, residents: $24 \%$, 49 seconds ( 13 seconds); fellows: $78 \%, 32$ seconds ( 13 seconds); and consultants: $86 \%, 25$ seconds ( 17 seconds). ${ }^{5}$ An increasing number of TI attempts were independently associated with TI-related adverse events with rates ranging from 20 to $39 \%^{3,6}$

Laryngeal mask airway (LMA) is an orally introduced supraglottic airway tube with an inflatable cuffed mask at one end that forms a low-pressure airtight seal around the laryngeal inlet. LMA ventilation, unlike ETTs, can be successfully inserted blindly with greater ease and speed (average time for insertion $<10$ seconds). ${ }^{7}$ The procedure is relatively independent of operator skills. LMA provides the advantage of achieving a stable artificial airway without the use of a laryngoscope and improves hemodynamic stability during insertion. The major disadvantages of first-generation LMA, although lesser than bag-mask ventilation, are the gastric insufflation and the inability to suction the airway. ${ }^{8}$ The problem of gastric insufflation can be overcome to a certain extent by the use of newer generation LMA such as supreme

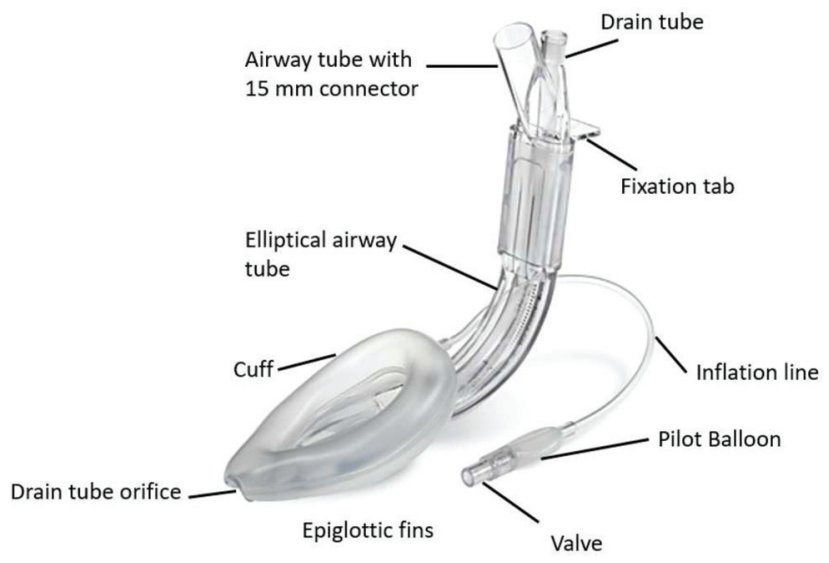

Fig. 1 Parts of supreme laryngeal mask airway.
LMA or i-gel LMA that minimizes gastric insufflation and provides access for gastric decompression ${ }^{9}$ ( - Fig. 1).

In 2015, Neonatal Resuscitation Program (NRP) recommended LMA as an alternative to the ETT in situations where the provider is "unable to intubate and unable to ventilate." LMA insertion is being taught in the NRP routinely. However, endotracheal intubation is the preferred method considered as the standard of care in neonatal resuscitation. ${ }^{10}$ First attempt success rates with LMA insertion was close to $100 \%$ in the previous studies. ${ }^{7,11,12}$

No recent study has evaluated the LMA insertion skills of neonatal resuscitation providers. Therefore, we wanted to study the proficiency of NRP certified providers in the technique of LMA insertion compared with the standard of care (ETT insertion). We also wanted to explore the provider's perception and confidence level in using LMA compared with ETT to secure an airway. We hypothesized that NRP certified providers would have LMA insertion skills equivalent to the standard of care (ETT insertion) and would be equally confident with both the skills.

\section{Materials and Methods}

\section{Study Site and Equipment}

A manikin-based study was performed after the approval of the Institutional Review Board at John R. Oishei Children's Hospital at Buffalo. The neonatal intensive care unit (NICU) at John R. Oishei Children's Hospital is the regional perinatal center for western New York, with more than 850 admissions out of more than 400 high-risk deliveries and 3,000 live births annually. Approximately one-third of these are transported from an outlying hospital. Our unit does more than 500 TIs per year. We used Nasco LF03623U infant airway management trainer withstand, standard size 1 LMA classic, and the uncuffed size of 3.0 ETT. The insertion of both the airway devices by each NRP certified provider was video recorded using an iPhone 7 plus (running iOS 12.3.1).

Participants

NRP certified providers with 1 or more years of experience since the first certification and with current valid NRP provider/instructor status were included in the study. We enrolled 31 NRP providers for this study. Providers with less than 1 year since first NRP certification or expired NRP certification status were excluded.

Study Design

The participants were instructed to insert an ETT and LMA in a manikin. The procedure was video recorded and was viewed later for data extraction. A single interpreter analyzed the data. The number of attempts needed for the successful placement of either device, time to insertion, and time to successful ventilation with a positive chest rise was recorded. 
We defined time to insert (seconds) as the time at which the provider introduced the laryngoscope blade/LMA device into the mouth until the correct positioning of either of the devices (including the removal of the stylet in ETT procedure and inflation of the cuff in LMA procedure). We defined time to ventilation (seconds) as the time from the introduction of laryngoscope until the delivery of the first positive pressure breath, which effectively inflated both the lungs. We defined failure to establish an airway as a failure to insert the device in two attempts successfully. After the completion of the procedures with the manikin, a five-point questionnaire was filled by each of the participants to assess their level of confidence in the skill, perception of ease of the procedure, and the ability to provide effective positive pressure ventilation (PPV).

\section{Statistical Methods}

\section{Sample Size}

We used the software program "PS: Power and Sample Size Calculation" version 3.1.6, October 2018, for sample size calculation. ${ }^{13}$ If the true difference in the mean time to start effective ventilation between ETT and LMA is 30 seconds, we needed to study at least 20 pairs of subjects to reject the null hypothesis that this response difference is zero with probability (power) 0.8 . The Type I error probability associated with this test of this null hypothesis is 0.05 .

\section{Data Analysis}

We used paired $t$-tests for the analysis of time to insertion/ventilation, the chi-square test for the analysis of the failure of the insertion of the airway devices, and the Kruskal-Wallis' test for the Likert scale questionnaire.

\section{Results}

Thirty-one providers participated in the study. Of the 31 participants, there were 3 neonatologists, 5 neonatology fellows, 5 pediatric residents in their second or third year of training, 14 advanced practice providers (neonatal nurse practitioners and physician assistants), and 4 registered respiratory therapists. There were $11 \mathrm{NRP}$ instructors in this cohort (-Table 1).

The mean duration of experience in the neonatal resuscitation was $11 \pm 10$ years, and mean period since the last NRP certification was $10 \pm 7$ months.

\section{LMA Experience}

There were 18 (58\%) providers who had hands-on exposure to LMA insertion during their NRP course, which includes 3 (38\%) neonatal fellows/attendings, 3 (60\%) pediatric residents, and 12 (66\%) advanced practice providers. Only four (13\%) providers ever had a chance to insert LMA in an infant in their clinical practice and none in the last 12 months before the study participation.

\section{ETT Experience}

There were 15 (48\%) providers who had more than 45 to 60 TIs in their clinical practice. This includes eight neonatal

Table 1 Participant characteristics of the study

Characteristic

1. Total number of providers participated in the study $(n)$

2. Mean duration of experience as NRP provider (y)

3. Mean duration since the last NRP course (mo)

4. Number of providers who were also NRP instructors

5. Number of providers who had hands-on exposure to LMA insertion in NRP

6. Number of providers who had at least one LMA insertion ever in an infant

7. Number of providers who had at least 45-60 ETT insertions in their clinical practice

Abbreviations: ETT, endotracheal tube; LMA, laryngeal mask airway; NRP, Neonatal Resuscitation Program; PPV, positive pressure ventilation.

fellows/attendings and seven advanced practice providers. Among them, nine (29\%) providers, which include four neonatal fellow/attendings and five advanced practice providers, had done more than 100 neonatal TIs. Nine (29\%) providers had less than 15 intubations in their careers so far, including five pediatric residents and four advanced practice providers.

\section{Comparison of LMA and ETT Insertion}

Eight out of 31 (26\%) providers had a failure to insert any one of the devices. The data from the remaining 23 (74\%) providers were analyzed for the time to insertion and ventilation (-Table 2).

Time to insertion: We found that the mean duration taken to insert the ETT and LMA was not statistically different (32 vs. 36 seconds). The mean (SD) time to insertion for ETT versus LMA among the neonatal physicians was 22 (10) versus 37 (18) seconds, pediatric residents was 57 (18) versus 23 (9) seconds, and advanced practice providers 28 (18) versus 41 (22) seconds. Based on the level of experience, providers who had neonatal resuscitation experience lesser than the sample median of 7 years had a mean time to insertion 34 (21) versus 42 (20) seconds. Providers with expertise higher than 7 years had a mean time to insertion 29 (19) versus 31 (19) seconds. All the subgroup analyses were statistically insignificant.

Time to ventilation: The mean time taken to provide the first positive pressure breath was also statistically similar (39 vs. 44 seconds) (see -Table 2 ). The mean (SD) time to ventilation for ETT versus LMA among the neonatal physicians was 29 (10) versus 44 (18) seconds, pediatric residents was 60 (25) versus 29 (6) seconds, advanced practice providers 38 (18) versus 49 (23) seconds. Based on the level of 


\begin{tabular}{|c|c|c|c|}
\hline Variable & $\begin{array}{l}\text { Endotracheal } \\
\text { tube }(N=23)\end{array}$ & $\begin{array}{l}\text { Laryngeal mask } \\
\text { airway }(N=23)\end{array}$ & $p$-Value \\
\hline $\begin{array}{l}\text { Mean time to insert in s } \\
\text { (95\% confidence interval) }\end{array}$ & $32(23-40)$ & $36(28-45)$ & 0.47 \\
\hline $\begin{array}{l}\text { Mean time to start ventilation in s } \\
\text { (95\% confidence interval) }\end{array}$ & $39(31-47)$ & $44(36-53)$ & 0.45 \\
\hline $\begin{array}{l}\text { Unsuccessful insertion } \\
\text { (percentage of total } 31 \text { participants) }\end{array}$ & $1(3.2)$ & 4 (12.9) & 0.16 \\
\hline $\begin{array}{l}\text { Successful insertion requiring }>2 \text { attempts } \\
\text { (percentage of total } 31 \text { participants) }\end{array}$ & $0(0)$ & $3(9.7)$ & 0.19 \\
\hline $\begin{array}{l}\text { Failure of insertion of airway device } \\
\text { (percentage of the total of } 31 \text { participants) }\end{array}$ & $1(3.2)$ & 7 (22.6) & $0.02^{\mathrm{a}}$ \\
\hline
\end{tabular}

Abbreviations: ETT, endotracheal tube; LMA, laryngeal mask airway. ${ }^{a} p$-Value $<0.05$.

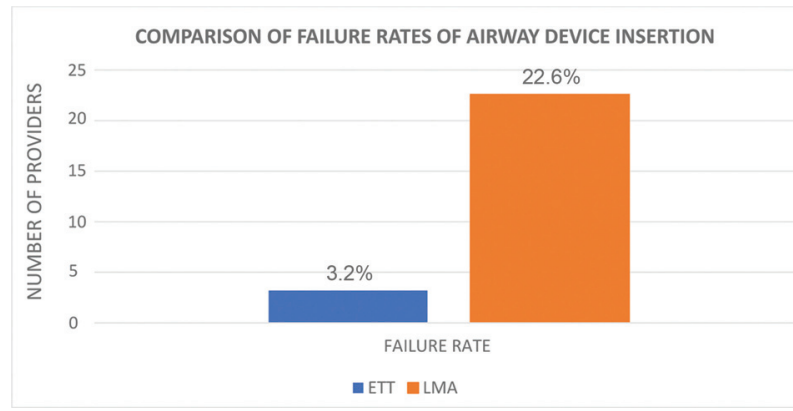

Fig. 2 Comparison of the failure rates of ETT and LMA insertion among 31 participants (1 vs. 7). ETT, endotracheal tube; LMA, laryngeal mask airway.

experience, providers who had expertise lesser than the sample median of 7 years had a mean duration of insertion 40 (21) versus 47 (20) seconds. Providers with an experience of more than 7 years had a mean duration of insertion of 38 (19) versus 41(23) seconds. All the subgroup analyses were statistically insignificant.

Frequency of failure rates: Among the 31 participants in the study, 5 providers were unable to insert either one of the airway devices ( 1 with ETT and 4 with LMA). Three providers needed more than two attempts to insert the LMA. None required more than two attempts to insert the ETT. A total of 7 out of 31 (23\%) providers met our definition of failure to place the LMA compared with 1 out of 31 (3\%) with the ETT. LMA insertion had a significantly higher failure rate than the standard of care ( 3 vs. $23 \%, p=0.02$ ) (see - Fig. 2 ). The providers who had a failure of ETT/LMA insertion included 25\% (2/8) neonatal fellows/attendings, 20\% (1/5) pediatric resident, and 28\% (5/18) advanced practice providers with no statistical difference based on the level of experience or the health care role.

Provider's perception questionnaire: In the analysis of the five-point Likert scale questionnaire (-Table 3), 12.9\% (4/31) of the participants felt confident to insert an LMA as against $64.5 \%(20 / 31)$ for TI $(p=0.000)$ (see - Fig. 3$) ; 29 \%$ (9/31) of the participants felt that LMA insertion is a more effortless procedure compared with $38.7 \%$ (12/31) for $\mathrm{TI} ; 41.9 \%$ (13/31) of the participants felt that using the LMA device is easy to provide effective PPV in neonates compared with $77.4 \%$ $(24 / 31)(p=0.005) ; 41.9 \%$ (13/31) of the respondents said they are less likely to use LMA as an alternative airway device in infants (see -Fig. 4).

\section{Discussion}

LMA use for PPV is a low-frequency emergency procedure in routine neonatal practice. Studies have established relatively easier skill acquisition and skill maintenance for both novice and experienced providers in various specialties, including neonatology. ${ }^{7,14,15}$ We anticipated that the providers would be equally skilled with LMA and ETT placement. However, we observed a higher failure rate for LMA insertion compared with ETT insertion. The providers took a relatively longer time to insert the LMA device compared with their ETT insertion. Many of the previously published studies that investigated the LMA insertion skill had an educational/practice component before the assessment. ${ }^{16,17}$ We wanted to assess the baseline preparedness of the NRP providers in the LMA use, so we did not include the procedural training before our study. We

\begin{tabular}{|c|c|c|c|}
\hline Variable & $\begin{array}{l}\text { Endotracheal tube }(N=31) \\
\text { Mean (SD) }\end{array}$ & $\begin{array}{l}\text { Laryngeal mask airway }(N=31) \\
\text { Mean (SD) }\end{array}$ & p-Value \\
\hline Confidence to insert the airway device & $3.8(1.2)$ & $2.4(1.1)$ & $0.000^{\mathrm{a}}$ \\
\hline Perception of ease of insertion & $3.2(0.96)$ & $3.1(1)$ & 0.301 \\
\hline Perception of ability to provide effective PPV & $4.1(0.81)$ & $3.5(0.85)$ & $0.005^{\mathrm{a}}$ \\
\hline
\end{tabular}

Abbreviations: PPV, positive pressure ventilation; SD, standard deviation.

a $p$-Value $<0.05$. 


\section{CONFIDENCE IN INSERTION OF AIRWAY DEVICE}

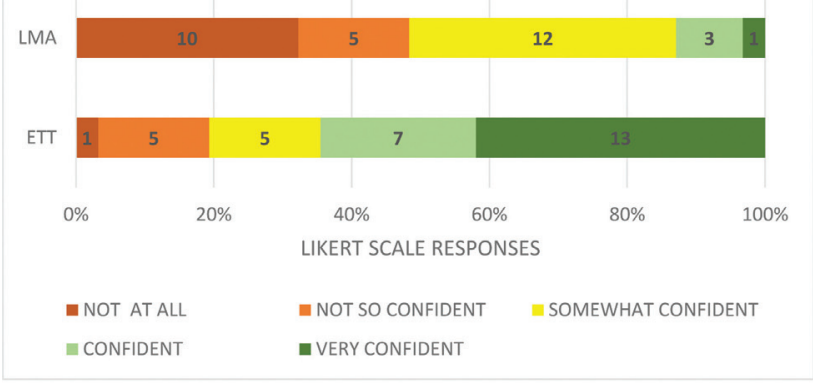

PERCEPTION OF EASE OF INSERTION

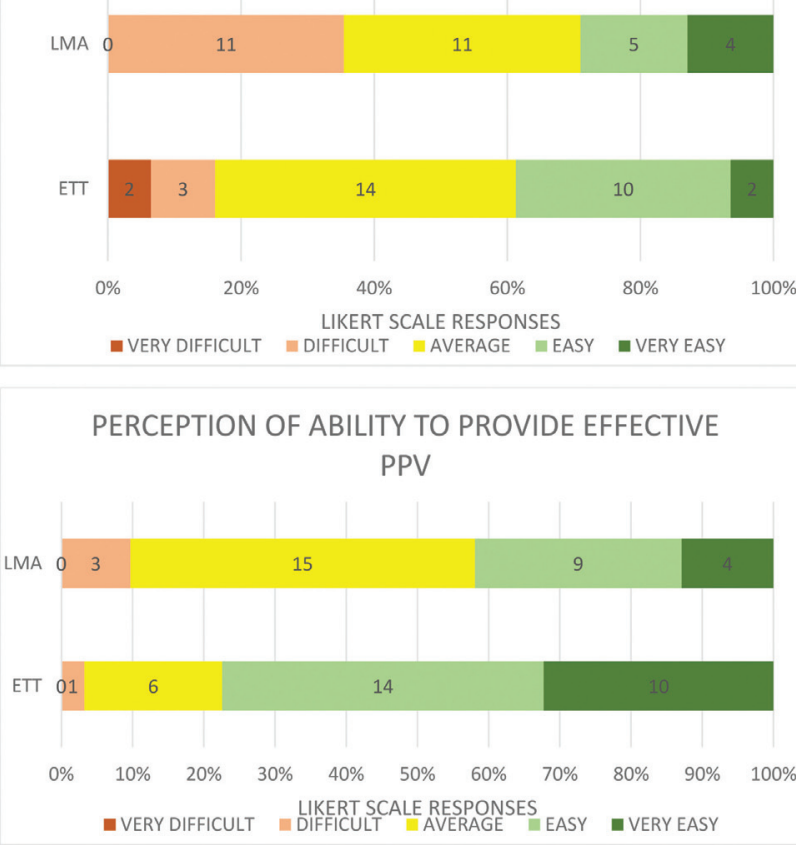

Fig. 3 A total of $100 \%$ stacked bar chart showing the likert scale responses of 31 participants for the confidence to insert the airway, perception of ease of insertion and the ability to provide effective PPV. ETT, endotracheal tube; LMA, laryngeal mask airway; PPV, positive pressure ventilation.

expected that the perception of the neonatal providers regarding airway management with LMA to be congruent with the current research. ${ }^{18-21}$ But participant NRP providers of this study felt that the LMA insertion is as sophisticated as TI with doubts about its ability to provide effective PPV.

We speculate that, generally, LMA insertion is not stressed enough during NRP training. In addition, LMA insertion being a low-frequency procedure in the current neonatal practice; even the experienced providers do not have adequate opportunities for developing this skill. Moreover, their experience-driven ETT insertion skill acquisition and maintenance shape their perception of LMA use in neonatology. The lack of proficiency in LMA insertion may not hinder the delivery of neonatal resuscitation in settings with the availability of experienced providers because they had more opportunities to get better at ETT insertion during their careers.

The neonatal practice has moved toward limiting the use of invasive ventilation after understanding the effects of barotrauma and volume trauma, especially in preterm infants. ${ }^{22-24}$ Nasal CPAP, nasal intermittent PPV, and heated humidified high-flow nasal cannula have become preferred modalities of respiratory support in the NICUs around the globe. ${ }^{25,26}$ This shift in practice can pose challenges for the early career neonatal health care providers in this era of noninvasive ventilation compounded by the factor of regionalization of newborn care. They may not have enough opportunities to acquire and maintain the relatively difficult ETT insertion skill, especially in levels 1 and 2 centers where invasive ventilation is out of their scope of clinical practice. The need for the use of airway devices infrequently arises in those levels 1 and 2 centers during childbirth. Intubation rates in community hospitals have shown a declining trend, a $50 \%$ reduction from 2010 to 2014 in one of the recent studies. ${ }^{27}$ Besides, NRP no longer recommends routine endotracheal suctioning in depressed infants born through meconium, which further limits the number of TIs in these more recently trained providers. In a situation where a need to secure an airway arises unexpectedly, the providers can be ill equipped with their skills to stabilize the airway of the infants prolonging the resuscitation resulting in avoidable complications and long-term morbidities.

Including the training of LMA insertion in the same way, as bag-mask ventilation and TI can mitigate the challenges that may arise for recently trained NRP providers, especially those practice outside the tertiary level neonatal centers. The current evidence should guide the teaching to impart knowledge and skills in an unbiased manner so that the providers' perception and confidence to use LMA in the appropriate settings are improved.

In the COVID-19 pandemic context, TI carries the highest risk as an aerosol-generating procedure (AGP) for the health care providers. ${ }^{28}$ They pose a danger to the safety of health care staff. Experience from adult medicine has shown that the use of second-generation LMA can have an essential role in critically ill patients' airway management. Supraglottic airway devices are recommended to be used instead of bagmask ventilation before establishing a definitive artificial airway. It provides the advantage of cautious use of available personnel by reducing the odds of exposure to AGPs. ${ }^{29} \mathrm{We}$ can utilize these advantages of LMA only if the providers have the proficiency and confidence to use it according to the current neonatal resuscitation recommendations.

Our study had a few limitations. It was a single-center study in a level $4 \mathrm{NICU}$, so the results cannot be generalized to other centers. A significant proportion of the participants in this study were experienced providers. They had better ETT insertion skills, which could have biased their perceptions. Results could be different if we do a similar investigation with early-career providers or providers predominantly practicing in levels 1 and centers. Our study did not have any training components at the start because we aimed to compare the baseline preparedness among the NRP 


\section{LMA AS AN ALTERNATIVE AIRWAY DEVICE}

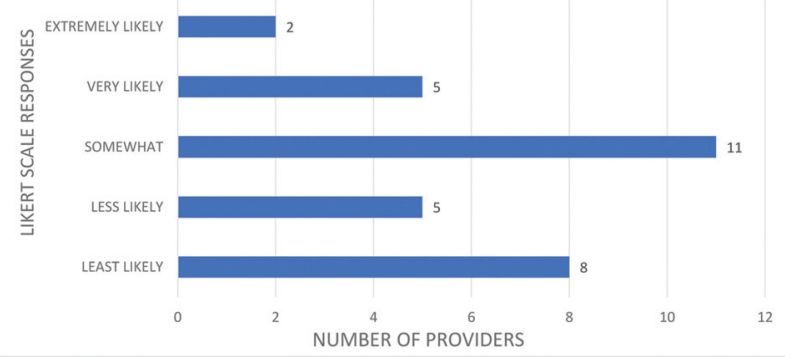

Fig. 4 Bar chart showing the Likert scale responses for likelihood of LMA use as an alternative airway device in neonates. LMA, laryngeal mask airway.

providers. Lack of an educational element could have impacted our study results. Follow-up on our study with a demonstration of the LMA insertion using an NRP developed video (https://youtu.be/TPBQd35EVgk) to all the study participants is underway. All the participants would retake the Likert scale questionnaire. We aim to determine if such a simple virtual refresher would alter the procedure's perceptions among the NRP providers.

Future studies, including NRP providers engaged in various levels of neonatal care, are necessary. Analysis of a larger stratified sample, including providers from levels 1 and 2 nurseries in the community hospitals apart from the higher level NICUs in the teaching hospitals, would help us better understand the knowledge gaps and changes necessary in skills training.

\section{Conclusion}

The NRP certified providers in this study did not demonstrate proficiency in LMA skills equivalent to the endotracheal intubation (standard of care). This study shows that there is scope for improving the LMA insertion training in the Neonatal Resuscitation Program.

\section{Authors' Contributions}

S.M. contributed to conceptualization, methodology, formal analysis, investigation, and writing the original draft. M.R. contributed to conceptualization, supervision, reviewing, and editing of the manuscript.

\section{Conflict of Interest}

M.R. reports grants from American Academy of Pediatrics -NRP Research Grant, outside the submitted work. S.M. reports grants from American Academy of PediatricsNeonatal Resuscitation Program Young Investigator Award, outside the submitted work.

\section{Acknowledgment}

The authors thank Praveen Chandrasekharan, MD (Assistant Professor of Pediatrics, University at Buffalo) for helping with the statistical analysis.

\section{References}

1 Niermeyer S. From the Neonatal Resuscitation Program to helping babies breathe: global impact of educational programs in neonatal resuscitation. Semin Fetal Neonatal Med 2015;20(05): 300-308

2 Andreatta PB, Dooley-Hash SL, Klotz JJ, Hauptman JG, Biddinger B, House JB. Retention curves for pediatric and neonatal intubation skills after simulation-based training. Pediatr Emerg Care 2016; 32(02):71-76

3 Herrick HM, Glass KM, Johnston LC, et al. for the NEAR4NEOS Investigators. Comparison of neonatal intubation practice and outcomes between the neonatal intensive care unit and delivery room. Neonatology 2020;117(01):65-72

4 Haubner LY, Barry JS, Johnston LC, et al. Neonatal intubation performance: room for improvement in tertiary neonatal intensive care units. Resuscitation 2013;84(10):1359-1364

5 O'Donnell CP, Kamlin CO, Davis PG, Morley CJ. Endotracheal intubation attempts during neonatal resuscitation: success rates, duration, and adverse effects. Pediatrics 2006;117(01):e16-e21

6 Hatch LD, Grubb PH, Lea AS, et al. Endotracheal intubation in neonates: a prospective study of adverse safety events in 162 infants. J Pediatr 2016;168:62-6.e6

7 Paterson SJ, Byrne PJ, Molesky MG, Seal RF, Finucane BT. Neonatal resuscitation using the laryngeal mask airway. Anesthesiology 1994;80(06):1248-1253, discussion 27A

8 Trevisanuto D, Micaglio M, Ferrarese P, Zanardo V. The laryngeal mask airway: potential applications in neonates. Arch Dis Child Fetal Neonatal Ed 2004;89(06):F485-F489

9 Verghese C, Ramaswamy B. LMA-Supreme-a new single-use LMA with gastric access: a report on its clinical efficacy. $\mathrm{Br} J$ Anaesth 2008;101(03):405-410

10 Kattwinkel J, Perlman JM, Aziz K, et al. Part 15: neonatal resuscitation: 2010 American Heart Association guidelines for cardiopulmonary resuscitation and emergency cardiovascular care. Circulation 2010;122(18, Suppl 3):S909-S919

11 Gandini D, Brimacombe JR. Neonatal resuscitation with the laryngeal mask airway in normal and low birth weight infants. Anesth Analg 1999;89(03):642-643

12 Trevisanuto D, Micaglio M, Pitton M, Magarotto M, Piva D, Zanardo V. Laryngeal mask airway: is the management of neonates requiring positive pressure ventilation at birth changing? Resuscitation 2004;62(02):151-157

13 Dupont WD, Plummer WD Jr. Power and sample size calculations for studies involving linear regression. Control Clin Trials 1998;19 (06):589-601

14 Yang C, Zhu X, Lin W, et al. Randomized, controlled trial comparing laryngeal mask versus endotracheal intubation during neonatal resuscitation-a secondary publication. BMC Pediatr 2016; 16(01):17

15 Morley CJ, Davis PG, Doyle LW, Brion LP, Hascoet JM, Carlin JBCOIN Trial Investigators. Nasal CPAP or intubation at birth for very preterm infants. N Engl J Med 2008;358(07):700-708

16 Westhoff M, Schönhofer B, Neumann P, et al. [Noninvasive mechanical ventilation in acute respiratory failure [in German]. Pneumologie 2015;69(12):719-756

17 Gandini D, Brimacombe J. Manikin training for neonatal resuscitation with the laryngeal mask airway. Paediatr Anaesth 2004;14 (06):493-494

18 Weksler N, Tarnopolski A, Klein M, et al. Insertion of the endotracheal tube, laryngeal mask airway and oesophageal-tracheal Combitube. A 6-month comparative prospective study of acquisition and retention skills by medical students. Eur J Anaesthesiol 2005;22(05):337-340

19 Trevisanuto D, Parotto M, Doglioni N, Ori C, Zanardo V, Micaglio M. The Supreme Laryngeal Mask Airway ${ }^{\mathrm{TM}}$ (LMA): a new neonatal supraglottic device: comparison with Classic and ProSeal LMA in a manikin. Resuscitation 2012;83(01):97-100 
20 Roberts I, Allsop P, Dickinson M, Curry P, Eastwick-Field P, Eyre G. Airway management training using the laryngeal mask airway: a comparison of two different training programmes. Resuscitation 1997;33(03):211-214

21 Bansal SC, Caoci S, Dempsey E, Trevisanuto D, Roehr CC. The laryngeal mask airway and its use in neonatal resuscitation: a critical review of where we are in 2017/2018. Neonatology 2018 ; 113(02):152-161

22 Trevisanuto D, Cavallin F, Nguyen LN, et al. Supreme laryngeal mask airway versus face mask during neonatal resuscitation: a randomized controlled trial. J Pediatr 2015;167(02): 286-91.e1

23 Pejovic NJ, Trevisanuto D, Lubulwa C, et al. Neonatal resuscitation using a laryngeal mask airway: a randomised trial in Uganda. Arch Dis Child 2018;103(03):255-260

24 Finer NN, Carlo WA, Walsh MC, et al. SUPPORT Study Group of the Eunice Kennedy Shriver NICHD Neonatal Research Network. Early
CPAP versus surfactant in extremely preterm infants. N Engl J Med 2010;362(21):1970-1979

25 Behnke J, Lemyre B, Czernik C, Zimmer K-P, Ehrhardt H, Waitz M. Non-invasive ventilation in neonatology. Dtsch Arztebl Int 2019; 116(11):177-183

26 Roberts CT, Hodgson KA. Nasal high flow treatment in preterm infants. Matern Health Neonatol Perinatol 2017;3:15

27 Marx A, Arnemann C, Horton RL, Amon K, Joseph N, Carlson J. Decreasing neonatal intubation rates: trends at a community hospital. J Neonatal Nurs 2016;22(05):231-235

28 Tran K, Cimon K, Severn M, Pessoa-Silva CL, Conly J. Aerosol generating procedures and risk of transmission of acute respiratory infections to healthcare workers: a systematic review. PLoS One 2012;7(04):e35797

29 Cheung JC, Ho LT, Cheng JV, Cham EYK, Lam KN. Staff safety during emergency airway management for COVID-19 in Hong Kong. Lancet Respir Med 2020;8(04):e19 